\title{
Pure neural leprosy—mind the diagnosis
}

\author{
Wim Brandsma ${ }^{\mathrm{a}}$, Erik Post ${ }^{\mathrm{b}}$, Inge Wagenaar ${ }^{\mathrm{c}}$, Khorshed Alam ${ }^{\mathrm{d},}{ }^{\dagger}$, \\ Vanaja Shetty ${ }^{\mathrm{e}}, \dagger$, Sajid Husain ${ }^{\mathrm{f}}{ }^{\dagger}$, Cita Rosita Sigit Prakoeswa ${ }^{\mathrm{g},}{ }^{\dagger}$, \\ Mahesh Shah ${ }^{\text {h, }} \uparrow \&$ Krishna Bahadur Tamang ${ }^{\mathrm{i},} \dagger$ \\ ${ }^{a}$ Retired, consultant, leprosy - hand rehabilitation, Green Pastures Hospital \\ and Rehabilitation Center, International Nepal Fellowship, P.O. Box 5, \\ Pokhara, Nepal \\ ${ }^{\mathrm{b}}$ For Tenlep study: Sr. Advisor Health, Royal Tropical Institute, Amsterdam, \\ The Netherlands \\ ${ }^{\mathrm{c}}$ Centre for Infectious Disease Control, National Institute of Public Health \\ and the Environment (RIVM), Bilthoven, The Netherlands \\ ${ }^{\mathrm{d}}$ Rural Health Program, The Leprosy Mission International Bangladesh, \\ Nilphamari, Bangladesh \\ e Foundation for Medical Research, Mumbai, India \\ ${ }^{\mathrm{f}} J A L M A$ institute of Leprosy \& Other Mycobacterial Diseases, Agra, India \\ ${ }^{\mathrm{g}}$ Dr. Soetomo Teaching Hospital - Universitas Airlangga, Surabaya, \\ Indonesia \\ ${ }^{\mathrm{h}}$ Anandaban Hospital and Mycobacterial Research Laboratories, The \\ Leprosy Mission Nepal, Kathmandu, Nepal \\ ${ }^{\mathrm{i}}$ Lalgadh Leprosy Hospital and Services Centre Dhanusha, Lalgadh, Nepal
}

Submitted 8 October 2020; Accepted 12 January 2021

\begin{abstract}
Summary
Introduction: Pure Neural Leprosy (PNL) is a known clinical diagnosis of leprosy. There is great epidemiological variability in the diagnosis and there appears to be no consensus about its definition and classification. Given the reported prevalence it is for operational reasons important that there is consensus about its definition, classification and treatment.

Method: The TENLEP (Treatment of early Neuropathy in LEProsy) database was used to analyze patients that were enrolled with a diagnosis of PNL to determine proportions of patients with PNL enrolled in six research sites in four geographically different leprosy endemic countries. Demographics were looked at and frequency of nerves involved in PNL.

The database consists of data of 1240 patients that were enrolled to determine efficacy of prednisolone in the prevention of clinical neuropathy $(N=372)$ or the treatment of clinical neuropathy $(N=868)$.
\end{abstract}

\footnotetext{
Correspondence to: W. Brandsma, Retired, consultant, leprosy - hand rehabilitation, Green Pastures Hospital and Rehabilitation Center, International Nepal Fellowship, P.O. Box 5 Pokhara, Nepal (e-mail: jwbrandsma@gmail.com)

$\dagger$ Study sites PIs.
} 
Results: In the clinical trial $76(8.8 \%)$, and in the subclinical trial $28(7.5 \%)$ patients were enrolled with the diagnosis of PNL. There was great variability in diagnosis and enrollment of PNL patients in the two cohorts. The ulnar nerve is the most affected (enlargement and/or function loss) of all nerves.

Conclusion: Our findings corroborate with the findings of other studies regarding the occurrence of PNL. The diagnosis needs to be carefully made and additional tests or assessments may be needed before a definitive diagnosis of Pure Neural Leprosy can be made.

Keywords: Diagnosis, epidemiology, diagnostic test, neuropathy, classificaion

\section{Introduction}

In most leprosy patients, the diagnosis is based on one or more of the three classical cardinal signs: hypesthesia in a skin lesion (compared with the contralateral site), palpable nerve enlargement or a positive skin smear for M. Leprae.

The diagnosis of (pure) neural leprosy (PNL) is commonly based only on palpable nerve enlargement and/or nerve tenderness, often accompanied by nerve function loss e.g. weakness or paralysis and loss of sensation. Sometimes only paresthesia or numbness is reported.

Pure neural leprosy is recognized as a separate clinical leprosy manifestation. The incidence of neural leprosy is variously reported to be between 5.5 and $17.7 \% .^{1}$ In a defined cohort of paucibacillary (PB) leprosy, Talwar found 42 patients (10.7\%) diagnosed as PNL. ${ }^{2}$ All patients were smear negative.

Kumar reported PNL in $65(4.2 \%)$ of 1542 patients. $^{3}$ Mononeuritis was seen in $40 \%$ of patients and the right ulnar and common peroneal nerve were the most commonly involved nerves. Also in this study skin smears were negative in all the subjects.

In a recent review article Kumar estimates PNL to be in the range of 4-8\% for India. ${ }^{4}$

In a prospective study from Brazil, 24 PNL were followed. ${ }^{5}$ In this study all patients were also smear negative. Paresthesia at the time of diagnosis was the main complaint. Ulnar motor and median sensory nerves were most impaired.

Van Brakel et al., reported 8.7\% patients with PNL in a cohort of 703 newly diagnosed patients in Nepal. ${ }^{6}$

In a recent review paper, the importance of diagnosing neural leprosy was emphasized. ${ }^{7}$ Interestingly, the article mentioned that when skin biopsies were performed in the territory of the affected nerves, many skin biopsies demonstrated leprosy pathology. Suneetha et al., reported $32.1 \%$ histopathological changes in apparently normal looking skin. ${ }^{8,}{ }^{9}$ Rao et al., also mentioned that in some studies skin lesions had developed post diagnosis. ${ }^{10}$

The main objective of this study is to describe the demographics, prevalence, and nerve involvement in pure neural leprosy from a large cohort of patients in six differently organized leprosy projects in four leprosy endemic countries: India, Nepal, Bangladesh and Indonesia.

A secondary objective is to alert leprosy workers to the possibility of a diagnosis of PNL following careful examination and investigations and not to misdiagnose leprosy when other neuromuscular pathology may be present. 


\section{Method and study population}

The data on neural leprosy in this study are based on the data from the Treatment of Early Neuropathy in LEProsy trials (TENLEP). These two, triple blinded-randomized clinical trials, assessed the efficacy of prednisolone in treating early clinical neuropathy or the prevention of neuropathy in subclinical neuropathy. ${ }^{11,12}$

Patients were assigned to the clinical trial based on Voluntary Muscles Testing (VMT) and sensory testing results using monofilaments (MFT) that showed defined nerve function impairment (NFI). Patients assigned in the subclinical trial would have normal VMT and MFT and would be enrolled in the subclinical trial based on criteria for subclinical NFI as determined by electroneurophysiological assessments. ${ }^{11}$ Prior to enrollment reliability studies were conducted in all research sites. ${ }^{13}$

In the study, motor loss was defined as grade 4 or less on the MRC scale. Sensory loss was defined as 3 or more points loss for a single nerve. Five filaments were used on three test sites for each nerve that could be affected in the upper extremity (ulnar, median, and radial cutaneous nerves); four filaments were used for the foot (posterior tibial and sural nerves).

Patients in the subclinical trial would have normal VMT and ST but NFI was based on decreased warmth perception and impaired nerve conduction parameters.

For other inclusion and exclusion criteria and definitions used, we refer to the protocol paper. $^{11}$

In the TENLEP studies 1240 patients were enrolled, 868 in the clinical and 372 in the subclinical study. The records of subjects that were classified as neural leprosy were screened to see that they were not coded for the presence of skin lesions and that there were no other variables coded that would be incompatible with neural leprosy e.g. clinical nerve function impairment in the subclinical trial. Six centers in four leprosy endemic countries participated in the clinical trial, four of these also in the subclinical trial.

The Ridley-Jopling classification was used to classify leprosy. Neural leprosy was assigned its own code. Ethical approval for these studies was obtained from all project sites.

\section{Results}

In the clinical study 868 patients were enrolled, of whom 80 were diagnosed as having neural leprosy. In the subclinical trial 372 patients were enrolled, of whom 29 were diagnosed as having neural leprosy (Tables 1,2). Following careful checking of all files, three patients in the clinical and one in the subclinical trial were for different reasons eliminated from analysis e.g. because of misclassification.

In the subclinical neuropathy trial, the ulnar nerve was the most affected nerve (enlargement) followed by the sural nerve (Table 3 ).

In the clinical trial the ulnar nerve was again the nerve which most often showed enlargement. Enlargement of nerves showed a similar pattern between the two trials (Tables 3 and 4).

We looked at nerve function impairment in the clinical trial and found again that the ulnar nerve is the most frequently impaired (Table 5). No differentiation is made between sensory and motor impairment.

Clinicians know that there is no one-on-one relationship between findings on palpation of a nerve and NFI. Table 6 illustrates this for the ulnar nerve. We also looked for right versus left differences. For the ulnar nerve we found that the right side showed more NFI than the left, the reverse for the lateral popliteal nerve (Table 7). 
Table 1. Neural leprosy in the two TENLEP trials

\begin{tabular}{cccccccc}
\hline $\begin{array}{c}\text { Clinical neuropathy } \\
\text { (center) }\end{array}$ & Enrolled & $\begin{array}{c}\text { Neural } \\
\text { leprosy }\end{array}$ & $\%$ & $\begin{array}{c}\text { Subclinical } \\
\text { neuropathy }\end{array}$ & Enrolled & $\begin{array}{c}\text { Neural } \\
\text { leprosy }\end{array}$ & $\%$ \\
\hline A & 82 & 9 & 11.0 & & 68 & 11 & 16.2 \\
B & 230 & 34 & 14.8 & & 138 & 17 & 12.3 \\
C & 232 & 6 & 2.6 & & 118 & 0 & - \\
D & 166 & 24 & 14.5 & & 48 & 0 & - \\
E & 101 & 1 & 1.0 & NA & - & - \\
F & 57 & 2 & 3.5 & NA & - & - \\
Total & 868 & 76 & 8.8 & 372 & 28 & 7.5 \\
\hline
\end{tabular}

NA. These two research sites did not participate in the subclinical neuropathy trial.

Table 2. Age and gender across the two trials

\begin{tabular}{ccccrc}
\hline $\begin{array}{c}\text { Clinical } \\
\text { neuropathy }\end{array}$ & $N$ & Age & $\begin{array}{l}\text { Subclinical } \\
\text { neuropathy }\end{array}$ & $N$ & Age \\
\hline Men & 66 & $32.1(16-60)$ & & 26 & $31.7(16-60)$ \\
Women & 10 & $37.5(17-45)$ & & 2 & $37.5(35-40)$ \\
\hline
\end{tabular}

Table 3. Number (\%) of nerves enlarged in PNL in the subclinical trial

\begin{tabular}{cccccccc}
\hline Subclinical trial & $\begin{array}{c}\text { Greater } \\
\text { auricular }\end{array}$ & Ulnar & Median & $\begin{array}{c}\text { Radial } \\
\text { cutaneous }\end{array}$ & $\begin{array}{c}\text { Lateral } \\
\text { popliteal }\end{array}$ & $\begin{array}{c}\text { Posterior } \\
\text { tibial }\end{array}$ & Sural \\
\hline$N=28$ (56 Nerves) & 9 & 29 & 7 & 17 & 17 & 6 & 19 \\
$\%$ & 16.1 & 51.8 & 12.5 & 30.4 & 30.4 & 10.7 & 33.9 \\
\hline
\end{tabular}

Table 4. Number (\%) of nerves enlarged in PNL in the clinical trial

\begin{tabular}{cccccccc}
\hline Clinical trial & $\begin{array}{c}\text { Greater } \\
\text { auricular }\end{array}$ & Ulnar & Median & $\begin{array}{c}\text { Radial } \\
\text { cutaneous }\end{array}$ & $\begin{array}{c}\text { Lateral } \\
\text { popliteal }\end{array}$ & $\begin{array}{c}\text { Posterior } \\
\text { tibial }\end{array}$ & Sural \\
\hline$N=76$ (152 Nerves) & 10 & 78 & 24 & 36 & 40 & 27 & 21 \\
$\%$ & 6.6 & $\mathbf{5 1 . 3}$ & 15.8 & 23.7 & 26.3 & 17.8 & 13.8 \\
\hline
\end{tabular}

Table 5. Nerve function impairment in clinical trial

\begin{tabular}{cccccccc}
\hline $\begin{array}{c}N=76 \\
152 \text { nerves }\end{array}$ & Facial & Ulnar & Median & $\begin{array}{c}\text { Radial } \\
\text { cutaneous }\end{array}$ & $\begin{array}{c}\text { Lateral } \\
\text { popliteal }\end{array}$ & $\begin{array}{c}\text { Posterior } \\
\text { tibial }\end{array}$ & Sural \\
\hline NFI & 0 & 82 & 42 & 48 & 21 & 55 & 55 \\
$\%$ & 0 & $\mathbf{5 3 . 6}$ & 27.6 & 31.2 & 13.8 & 36.2 & 36.2 \\
\hline
\end{tabular}

Table 6. Ulnar nerve. Relationship between enlargement and clinical nerve function impairment

\begin{tabular}{ccc}
\hline & $N=160$ nerves & $\%$ \\
\hline Enlarged and loss & 65 & 40.6 \\
Not enlarged no loss & 60 & 37.5 \\
Not enlarged but loss & 19 & 11.9 \\
Enlarged no loss & 16 & 10.0 \\
\hline
\end{tabular}


Table 7. Clinical impairment ulnar and lateral popliteal nerves $(N=76)$

\begin{tabular}{cllcrr}
\hline Ulnar nerve & $N$ & $\%$ & $\begin{array}{c}\text { Lateral } \\
\text { popliteal }\end{array}$ & $N$ & $\%$ \\
\hline Right & 26 & 34.2 & & 1 & 1.4 \\
Right and Left & 21 & 27.6 & 8 & 10.5 \\
Left & 14 & 18.4 & 8 & 10.5 \\
No impairment & 15 & 19.7 & 59 & 77.6 \\
\hline
\end{tabular}

\section{Discussion}

As reported in other studies, we also found that some patients diagnosed with PNL developed skin lesions following the diagnosis of 'neural' leprosy.

Pure neural leprosy is a recognized manifestation of leprosy. A first proven case of neural leprosy was reported by Jopling in the Lancet. ${ }^{14}$ This well-known leprologist made some important points as to why PNL is often not diagnosed: (1) Ignorance of early symptoms by patients, (2) lack of awareness or recognition of PNL by physicians and (3) insufficient means to diagnose PNL when suspected. The difference in PNL prevalence between the research sites may partly be explained by these factors.

Additionally, in the TENLEP research sites differences in program structure, integrated versus vertical leprosy programs with different levels of staff involved, medical specialists versus health workers, may also have contributed to differences in diagnosing PNL subjects. Two centers reported few patients with PNL in the clinical study; two reported none in the subclinical study (Table 1).

The two centers that diagnosed subclinical neuropathy in PNL were two referral centers with technical staff well-trained to assess leprosy neuropathy by assessing nerve conduction, prior to the TENLEP study. The two centers that did not diagnose subclinical PNL, but diagnosed clinical PNL, often had breakdowns of equipment and in one center only one of two assessment techniques were used to diagnose subclinical nerve function impairment. No doubt these factors, in addition to factors named above, also influenced enrollment of patients in the subclinical neuropathy trial.

Overall, the numbers of reported PNL subjects in our study are within the ranges reported in other studies, albeit with great differences between the centers as explained above. ${ }^{2,4,7}$

In the absence of additional confirmatory test results, the diagnosis of neural leprosy should be made with caution. Skin smears were mandatory in the TENLEP study but are usually negative in PNL leprosy. ${ }^{4,10}$ There remains, nerve palpation as the only cardinal sign and the interpretation of clinical symptoms such as paresthesia, numbness, and nerve pain. Nerve palpation is 'subjective' but it has been shown that it can be done reliably for some nerves. ${ }^{15}, 16$ However, expertise in nerve palpation and interpretation of findings is usually only available with those routinely palpating nerves, which was the case in every research site.

\section{DEVELOPMENT OF SKIN LESIONS POST DIAGNOSIS}

Suneetha et al., showed that symptoms of PNL could precede skin lesions. In their series of 182 patients $62 \%$ developed one or two skin lesions within two years of diagnosis. ${ }^{9,}, 10$

Post-diagnosis development of skin lesions has also been observed in our cohorts, but exact numbers cannot be given because of incomplete data recording. Development of skin lesion(s) begs the question if neural leprosy could be considered a pre-clinical state of leprosy. More patients may have developed skin lesions, but this may have happened unnoticed. 


\section{CLASSIFICATION AND TREATMENT}

The Ridley-Jopling classification does not include PNL and the WHO guide for the elimination of leprosy does not mention PNL. ${ }^{17}$ Notably, the most recent WHO guideline also does not mention PNL. ${ }^{18}$ In our studies PNL patients were classified as PB and MB and no 'pattern' could be identified in the (sub)clinical trial of number of nerves involved in relation to PB-MB classification. In the clinical trial $44 / 76$ patients were classified as $M B$, in the subclinical trial 9/19.

The International Leprosy Association also does not give guidance for PNL classification. ${ }^{19}$ Maybe in the subclinical trial the number of electroneurophysiologically impaired nerves could have been considered in decision making (not analyzed), in addition to the number of enlarged nerves. The clinical trial, enrolling patients with clinical NFI, also showed that the number of impaired nerves did not correlate with the classification. We believe that the overall clinical impression, nerves impaired or enlarged, tenderness, social context, including contacts, etc., by the physicians may have played a role in classifying the patient as PB or MB.

\section{DEFINING PNL}

Talwar diagnosed PNL if there was “....anesthetic skin area, weakness or wasting of muscles, or tingling sensation or pain, accompanied by nerve trunk thickening". ${ }^{2}$ In a later paper we see a more refined diagnostic definition “...thickened peripheral nerve trunk with sensory loss in the area of distribution, with or without associated motor paralysis in the absence of any skin patch...". 4

For clinical purposes we propose that PNL could be defined as enlarged and/or tender peripheral (incl. cutaneous) nerves with or without sensory and/or motor impairment in the distribution area of the nerves commonly involved in leprosy in the absence of typical skin lesions for leprosy and a negative skin smear.

Whether or not treatment should be initiated depends on e.g. nerve(s) involved, severity of impairment, leprosy contacts and possibility of additional detailed diagnostic testing (see later).

Regarding the naming of PNL, Primary Neural Leprosy would be a better choice than Pure Neural Leprosy which denotes exclusivity.

\section{DIFFERENTIAL DIAGNOSIS/NERVES IN PNL}

It should be noted that patients that seek treatment for nerve related symptoms may be diagnosed as PNL, especially in leprosy endemic countries. In contact examinations 'patients' may be diagnosed as having enlarged nerves and be symptom free. In such instances an expectant policy may be in place.

Personal note WB: A few years after I first got involved with leprosy patients (1970s) I developed enlarged, visible, greater auricular nerves. Some experienced physicians felt I should start treatment (dapsone) and that the radial cutaneous nerve should be biopsied. Biopsy result was negative. After few months enlargement of nerves was gone and I 'defaulted' from DDS. I know of several other leprosy workers having experienced the same: Subclinical neural leprosy, no functional impairments, not progressing to clinical leprosy or nerve function impairment.

Kumar found that the right ulnar and right common peroneal were the most affected in PNL. ${ }^{3}$ In our study we also found a predominance of right ulnar nerve impairment in the clinical study but not the right common peroneal nerve (Table 7). More research is needed to 
determine if extremity dominance effects the etiology of leprosy (inoculation leprosy through skin breakdown) or manifestations of leprosy neuropathy.

Commonly, only four nerves are routinely palpated in a clinical examination: Greater Auricular, Radial Cutaneous, Ulnar and Lateral Popliteal. The first two are mainly important from a diagnostic point of view. The latter two are also important from a clinical, disability prevention point of view. The Facial nerve is not palpated and palpation of Sural, Posterior Tibial, and Median need 'expertise' and are usually not practiced. To our knowledge, no reliability studies have been done on palpation findings for these nerves. Sural or Greater Auricular nerves were never found enlarged in isolation or in combination in the clinical or subclinical trial.

Diabetes is now quite common, often in the range of 5-10\% of the adult population, in countries in which leprosy is still endemic. There could potentially be confusion about a diagnosis of neural leprosy when diabetic neuropathy may be mistaken for leprosy. The two neuropathies, leprosy and diabetic neuropathy, could mask each other in the absence of cardinal leprosy signs. However, diabetic neuropathy would probably be expected in the more affluent population while leprosy neuropathy is predominantly still a disease more prevalent in the lower economic strata. When available and accessible, additional clinical tests are needed to make a definitive diagnosis. ${ }^{10}$ For a review of possible diseases that may be confused with leprosy we refer to Garbino. ${ }^{7}$

The pattern of nerve involvement in leprosy is known. If there is loss of nerve function, either motor or sensory, not related to nerves commonly involved in leprosy then the 'suspicion index' for other neuromuscular diseases should be raised and additional clinical testing should be considered. Alternatively, referral to a center where leprosy expertise is available, or a 'wait and see' policy could be adopted.

In recent years, high-resolution sonography has become a useful tool in the (differential) diagnosis of neurological diseases and as such in the confirmation of neural leprosy. ${ }^{4}$ However, the cost of equipment and the expertise needed limits its use to referral centers. Kumar discusses all possible tests that could be useful in the confirmation of (neural) leprosy. ${ }^{4}$ Most of these tests, however, are not useful 'in the field' where more than $95 \%$ of patients are diagnosed in low resource, leprosy endemic, countries. For research purposes, differential diagnosis, relating pathology to function, efficacy of drug treatment etc. ultrasonography is a promising tool in the hands of experts to investigate nerve enlargement and associated pathology. ${ }^{20-23}$ MRI is useful as well, be it that sonography is more efficient, user friendly and more economic. ${ }^{10}$ Kumar presents a useful flowchart as a tool in the diagnosis of PNL. ${ }^{4}$

\section{MISSING THE DIAGNOSIS OF LEPROSY AND MISDIAGNOSING LEPROSY}

Because of its possible chronic physical and social consequences, it is important that health staff in leprosy endemic countries know about the possibility of pure neural leprosy and start treatment in a timely manner, preferably only following additional supporting tests.

Alternatively, the diagnosis of neural leprosy should not be given when neural signs and symptoms are different from or more extensive than can be expected from the predictable and limited patterns of loss in leprosy neuropathy.

\section{LIMITATION OF THE STUDY}

The numbers identified as PNL do not reflect the true incidence of PNL because in this study inclusion and exclusion criteria could have excluded patients with neural leprosy e.g. age, distance from the research site, refusal to participate. Likewise, the number of PNL patients 
in this study may also have been influenced by the fact that in this study monofilaments were used to assess sensory impairment where in earlier studies ballpoint pen testing was used.

\section{Conclusion}

This study reports on a pre-selected group of leprosy patients. No generalizations should be made from the findings in this report. The main purpose of this article is to raise awareness of the possibility of the diagnosis of pure neural leprosy-not to miss the diagnosis and not to misdiagnose it either.

Key points based on this study and literature:

- Leprosy is always neural but not necessarily 'pure' neural. Hypesthesia in a skin lesion is neural.

- When there is NFI outside the territories of known 'leprosy nerves' then further neurological examination is recommended to exclude or confirm other neuromuscular diseases.

- Diabetic neuropathy can mask leprosy neuropathy, thus screening for diabetes is advised.

- Sonography is a useful complementary diagnostic tool to confirm or exclude PNL.

- Skin biopsies from the innervation territory of an 'enlarged or tender' nerve may show pathology.

- There is no direct relationship between palpable nerve enlargement and NFI.

- Patients may develop skin lesions post PNL diagnosis.

\section{Research staff at research sites}

Bangladesh: Dr. B Bowers, Kallyan Kumar Kunda, Anil Soren, Kolpona Roy, Khorshed Alam; India (Bombay): Gospi Capadia, Ashish Khodke, Kulbushan Pawar, Dr. Vivek Pai; India (JALMA): Mishra Nath Kameshwar, Bipin Kumar, Hemant Singh; Indonesia: Linda Astari. Bagus Haryo Kusumaputra, ${ }^{8}$ Irmadita Citrashanty, ${ }^{8}$ Sylvia Anggraeni, ${ }^{8}$ Menul Ayu Umborowati, ${ }^{8}$ Renata Mayangsari, ${ }^{8}$ Riski Haris Sasongko, Agustina, ${ }^{8}$ Asmahani Thohiroh, ${ }^{8}$ Nepal (Anandaban): Dr. Deanna Hagge, Kapil Neupane, Ram Babu Bista, ${ }^{9}$ Pawan Parajuli, ${ }^{9}$ Apsara Ghimiri ${ }^{9}$ Nepal (Lalgadh): Shyam Pariyar, Jeevan Thapa and Shankar Paudel.

\section{Acknowledgement}

We like to thank Dr. PN Nicholls, for development of the database and statistical advice 'along the way'.

\section{References}

1 Noordeen SK. Epidemiology of (poly)neuritic leprosy. Lepr India, 1972; 44: 91-96.

2 Talwar S, Jha PK, Tiwaari VD. Neuritic leprosy: epidemiology and therapeutic responsiveness. Lepr Rev, 1992; 63: 263-268.

3 Kumar B, Kaur I, Dogra S et al. Pure neuritic leprosy in India: An appraisal. Int J Lepr, 2004; 72: $284-290$.

4 Kumar B. Pure or primary neuritic leprosy (PNL). Lepr Rev, 2016; 87: 450-455.

5 Jardim MR, Illarramendi X, Osvaldo JM et al. Pure neural leprosy. Arq Neuropsiquiatr, 2007; 65(4A): 969-973.

6 Van Brakel WH, Soldenhoff R de, McDougall AC. The allocation of leprosy patients into paucibacillary and multibacillary groups taking into account the number of body areas affected skin, or skin and nerve lesions. Lepr Rev, 1992; 63: 231-246.

7 Garbino JA, Marques W, Barreto JA et al. Pure neural leprosy: systematic review. Arq Neuro-Psiquiatr, 2013; 71: 397-404, doi:10.1590/0004-282X20130046.

8 Suneetha S, Arunthathi S, Chandi S et al. Histological studies in primary neuritic leprosy - changes in apparent normal skin. Lepr Rev, 1998; 69: 351-357. 
9 Suneetha S, Sigamoni A, Kurian N et al. The development of cutaneous lesions during follow-up of patients with primary neuritic leprosy. Int J Dermatol, 2005; 44: 224-229.

10 Rao PN, Suneetha S. Pure neuritic leprosy: current status and relevance. Ind J Derm Vener Lepr, 2016; 82: 252-261.

11 Wagenaar I, Brandsma JW, Post E et al. Two randomized controlled trials to study the effectiveness of prednisolone treatment in prevention and restoring clinical nerve function loss in leprosy: the TENLEP study protocols. BMC Neurol, 2012; 12: 159, https://bmcneurol.biomedcentral.com/articles/10.1186/1471-2377-12159.

12 Wagenaar I, Post E, Brandsma JW et al. Effectiveness of 32 versus 20 weeks of prednisolone in leprosy patients with recent nerve function impairment: a randomized controlled trial. PloS Negl Trop Dis, 2017; https://www. ncbi.nlm.nih.gov/pmc/articles/PMC5643133/pdf/pntd.0005952.pdf.

13 Brandsma JW, Wagenaar IW, Post E et al. Reliability of clinical nerve function assessment in peripheral neuropathies. Lepr Rev, 2014; 85: 29-35.

14 Jopling WH, Morgan-Hughes JA. Pure neural tuberculoid leprosy. Br Med J, 1965; 2: 799-800.

15 Chen S, Wang Q, Chu T et al. Interobserver reliability in assessment of sensation of skin lesion and enlargement of peripheral nerves. Lepr Rev, 2006; 77: 371-376.

16 Kolappan C, Selvaraj R, Khudoos A et al. Repeatability of nerve thickness assessment in the clinical examination for leprosy. Lepr Rev, 1995; 66: 224-228.

17 World Health Organization. Guide to the Eliminating Leprosy as a Public Health Problem. 2nd ed, Geneva: WHO, 1997.

18 World Health Organization. Guidelines for the Diagnosis, Treatment and Prevention of Leprosy. Geneva: WHO, 2018.

19 International Leprosy Association - Technical Forum. Diagnosis and classification of leprosy. Lepr Rev, 2002; 73(June): S17-S26.

20 Bathala L, Krishnam N, Kumar HK et al. Extensive sonographic ulnar nerve enlargement above the medial epicondyle is a characteristic sign in Hansen's neuropathy. PLoS Negl Trop Dis, 2017; doi:10.1371/journal.pntd.0005766.

21 Chaduvula MV, Visser LH, Suneetha S et al. High-resolution sonography as an additional diagnostic and prognostic tool to monitor disease activity in leprosy. A two- year prospective study. Ultraschall Med, 2018; 39: 80-89, doi:10.1055/s-0042-108430.

22 Jain S, Visser LH, Praveen TLN et al. High-resolution sonography: a new technique to detect nerve damage in leprosy. PLoS Negl Trop Dis, 2009; 3(8): e498.

23 Jain S, Visser LH, Suneetha S. Imaging techniques in leprosy clinics. Clin Derm, 2016; 34: 70-78. 